\title{
The Role of Serum Galectin-3 Levels in Patients with Sarcoidosis
}

\author{
Kaan Kara ${ }^{a}$ Seda Tural Onur ${ }^{a}$ Sinem Nedime Sokucu ${ }^{a}$ Ozlem Kahyab \\ Cengiz Ozdemir ${ }^{a}$ Evin Ademoglu ${ }^{c}$
}

aDepartment of Chest Desease, Yedikule Chest Diseases and Chest Surgery Training and Research Hospital, Istanbul,

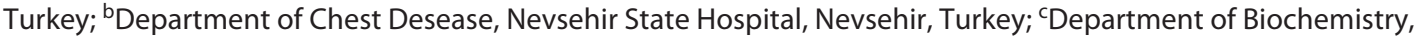
Istanbul Faculty of Medicine, Istanbul University, Istanbul, Turkey

\section{Highlights of the Study}

- Sarcoidosis is a systemic granulomatous disease with progressive fibrosis.

- Galectin-3 plays a crucial role in the stimulation of inflammation and autoimmune disorders.

- Levels of serum galectin-3 increase in the presence of diseases like sarcoidosis that progress with pulmonary fibrosis.

- Galectin-3 can be considered for use in determining the staging and severity of sarcoidosis.

\section{Keywords}

Sarcoidosis · Galectin-3 · Pulmonary fibrosis · Inflammation

\section{Abstract}

Introduction: Galectin-3 is a multifunctional protein, the levels of which increase in the presence of diseases that progress with pulmonary fibrosis. This study investigated the role of galectin-3 levels in the staging and assessing of the severity of sarcoidosis. Methods and Subjects: Seventy-three subjects were studied; 25 were healthy individuals and 48 patients had pathologically confirmed diagnosis of sarcoidosis in which other potential causes had been ruled out. Galectin-3 levels were measured and compared in terms of such parameters as hemogram, biochemistry, age, body mass index, and smoking status. Results: The mean galectin-3 levels of the sarcoidosis patients $(14.87 \pm 5.57)$ were significantly higher than those in the healthy subjects (11.81 \pm 2.67$)$, and the mean galectin-3 levels differed significantly among different stages of the disease $(p<0.05)$. The serum galectin-3 level in patients with stage 2,3 , and 4 sarcoidosis was found

karger@karger.com www.karger.com/mpp

Karger $\stackrel{\text { ' }}{5}$
(C) 2021 The Author(s)

Published by S. Karger AG, Basel

This is an Open Access article licensed under the Creative Common Attribution-NonCommercial-4.0 International License (CC BY-NC) (http://www.karger.com/Services/OpenAccessLicense), applicable to the online version of the article only. Usage and distribution for commercial purposes requires written permission. to be higher than in patients with stage 0 and 1 sarcoidosis and the control group. In addition, serum galectin-3 levels in the sarcoidosis patients had significant positive correlations with blood urea nitrogen, alkaline phosphatase, white blood cells, red blood cell, hemoglobin, and neutrophil levels (34.9\% [ $p<0.05] ; 40.1 \%[p<0.05] ; 41.2 \%$ [ $<<0.01] ; 43.3 \%$ [ $p$ $<0.01$ ]; 34.7\% [ $p<0.05$ ]; and 40.6\% [ $p<0.01$ ], respectively) and a significant negative correlation with the platelet distribution width levels $(p<0.05)$. Conclusion: Serum galectin-3 levels are significantly elevated in sarcoidosis patients with parenchymal involvement at stage 2 or higher, suggesting that serum galectin-3 levels can be used to estimate disease severity in sarcoidosis.

(c) 2021 The Author(s) Published by S. Karger AG, Basel

\section{Introduction}

Sarcoidosis is a systemic granulomatous disease with an unknown etiology that progresses with multi-systemic involvements. Its yearly incidence has been reported as 
$0.1-81$ per 100,000 and prevalence has been reported as $0.1-640$ per 100,000 . The prognosis and course of the disease may be consistent with its mode of onset and extent [1-3]. While various parameters for monitoring the course of the disease have been investigated, a specific biomarker has yet to be identified. Several parameters have been investigated to predict the activity of sarcoidosis, including angiotensin-converting enzyme and adenosine deaminase levels, lysozyme, neopterin, $\beta 2$ microglobulin, hypergammaglobulinemia, Krebs von den Lungen-6, chitotriosidas, fibronectin, collagenase, hyaluronan, and fibronectin levels $[4,5]$. Two studies have shown that the Krebs von den Lungen- 6 and chitotriosidas biomarkers can be used to determine the severity of sarcoidosis $[6,7]$.

Galectin-3 is a $\beta$-galactoside-binding animal lectin that has sites of carbohydrate recognition $[8,9]$. Galectin- 3 is released from the cytoplasm or nucleus of various cell types, including but not limited to, monocytes (MONs), macrophages, and endothelial, microglia, and epithelial cells [10]. Galectin-3 is a multifunctional protein that is involved in various physiological and pathologic processes, such as cell growth, angiogenesis, carcinogenesis, and inflammation. Extracellular galectin-3 mediates inflammation and leukocyte adhesion and plays roles in the retrieval and activation of neutrophils (NEUs) [11] and also has a crucial role in the stimulation of inflammation in a wide range of acute and chronic events, particularly neurologic degeneration, autoimmune disorders, atherosclerosis and heart failure, and response to infections or diseases of the lungs, kidney, and liver [12]. A recent study by Sun et al. [13] showed a relationship between galectin-3 level and prognosis of acute kidney injury. These observations suggest that galectin-3 may result in fibrosis in several systems.

Increased levels of serum galectin-3 in the presence of diseases progressing with pulmonary fibrosis have been reported [14]. Taking into account the limited number of studies investigating the relationship between galectin-3 levels and pulmonary diseases, some of which reported significant findings in the presence of pulmonary fibrosis, we hypothesized that serum galectin-3 may be used as a biomarker for the staging and assessment of severity of sarcoidosis. In the present study, we investigated the levels of galectin-3 in sarcoidosis patients and healthy controls.

\section{Subjects and Methods}

The present study included patients aged between 18 and 75 years who were followed in our hospital and diagnosed with pulmonary sarcoidosis after other potential causes were ruled out. The inducted patients had the following features: they had histo- pathological evidence of granuloma without calcification necrosis; they did not have interstitial lung disease other than sarcoidosis, or organ malignancy or history of chronic disorders. They made regular outpatient clinic visits and agreed to participate in the study. Healthy subjects aged between 18 and 75 years with no history of chronic disease and who voluntarily agreed to participate in the study constituted the control group. The study was initiated after approval was granted by the Ethics Committee of our hospital (Research Protocol Number 4221). The patients were informed about the study, and all signed an informed consent form.

The following data were garnered and evaluated for the $48 \mathrm{pa}-$ tients who agreed to participate in the study: demographic characteristics (age, gender, height, weight, and smoking status), pulmonary function test results, methods of diagnosis, current treatments, presence of extrapulmonary involvement, galectin-3 levels, and results of hemogram and biochemistry investigations performed within the last 6 months, obtained from hospital records.

The following data were recorded and evaluated for the 25 healthy controls who agreed to participate in the study: demographic characteristics (age, gender, height, weight, and smoking status), serum galectin-3 levels, as well as hemogram and biochemistry investigations performed within the last 6 months, obtained from hospital records. Galectin-3 levels of all sarcoidosis patients $(n=48)$ were compared along with their demographic data, pulmonary function test results, current treatments (if any), presence of extrapulmonary involvement, and hemogram and biochemistry investigations performed within the last 6 months, which were obtained from hospital records. Galectin-3 levels of all the healthy controls $(n=25)$ were compared along with their demographical data, body mass index (BMI), smoking status, and hemogram and biochemistry investigations performed within the last 6 months, obtained from hospital records.

Blood samples were obtained from both groups and centrifuged at $8,000 \mathrm{rpm}$ for $10 \mathrm{~min}$ at $4^{\circ} \mathrm{C}$. Serum samples were stored in Eppendorf tubes at -21 to $-40^{\circ} \mathrm{C}$. Galectin-3 levels in the serum samples were measured using a commercial kit based on the ELISA method (eBioscience, catalog no: BMS279/4, lot no: 133218001).

Based on the hemogram results of the peripheral blood, the NEU lymphocyte (LYM) ratio was calculated by dividing the absolute NEU count by the absolute LYM count, and the thrombocyte LYM ratio was calculated by dividing the absolute thrombocyte count by the absolute LYM count. Radiological classification was carried out by two different blinded pulmonologists, following the Scadding stage system, and classification difference between the specialists was detected using the Kappa method.

Numerical data were evaluated using the IBM SPSS Statistics 23 program, and measures of central tendency were presented as mean, standard deviation, minimum, and maximum values. Frequency distribution of the categorical variables was presented. An independent samples $t$ test was used to evaluate the differences between the 2 groups, and a one-way variance analysis (ANOVA) was used to assess the potential differences between more than 2 groups. Based on the results of the ANOVA, a Levene's test was applied to assess the homogeneity of variances, and a multiple comparison test (Bonferroni or Tamhane's $T^{2}$ ) was carried out to identify the group(s) causing significant difference. The differences between the groups were investigated with a Bonferroni test for variables with homogeneous variances and with Tamhane's $T^{2}$ test for variables without homogeneous variances. The Pearson Correlation Coefficient was used to investigate the relationship between serum galectin-3 levels, clinical characteristics, and blood parameters.
60

Med Princ Pract 2022;31:59-65

DOI: $10.1159 / 000521467$
Kara/Tural Onur/Nedime Sokucu/Kahya/ Ozdemir/Ademoglu 
Table 1. Investigation of differences in parameters (independent samples $t$ test)

\begin{tabular}{|c|c|c|c|c|c|}
\hline Parameters & $N$ & Mean & SD & $t$ & $p$ value \\
\hline \multicolumn{6}{|l|}{ Galectin-3 levels } \\
\hline Sarcoidosis patients & 48 & 14.87 & 5.567 & \multirow{2}{*}{3.171} & \multirow{2}{*}{$0.002^{* *}$} \\
\hline Healthy controls & 25 & 11.81 & 2.665 & & \\
\hline \multicolumn{6}{|l|}{ Age } \\
\hline Sarcoidosis patients & 48 & 45.21 & 11.900 & \multirow{2}{*}{3.166} & \multirow{2}{*}{$0.002 * *$} \\
\hline Healthy controls & 25 & 36.16 & 10.953 & & \\
\hline \multicolumn{6}{|l|}{ BMI } \\
\hline Sarcoidosis patients & 48 & 27.27 & 5.476 & \multirow{3}{*}{2.144} & \multirow{3}{*}{$0.035 *$} \\
\hline Healthy controls & 25 & 24.76 & 2.827 & & \\
\hline Healthy controls & 25 & 9.2 & 12.261 & & \\
\hline \multicolumn{6}{|l|}{ BUN } \\
\hline Sarcoidosis patients & 46 & 29.33 & 6.415 & \multirow{2}{*}{3.371} & \multirow{2}{*}{$0.001 * *$} \\
\hline Healthy controls & 20 & 23.70 & 5.768 & & \\
\hline \multicolumn{6}{|l|}{ Creatinine } \\
\hline Sarcoidosis patients & 45 & 0.76 & 0.164 & \multirow{2}{*}{0.659} & \multirow{2}{*}{0.513} \\
\hline Healthy controls & 21 & 0.73 & 0.181 & & \\
\hline \multicolumn{6}{|l|}{ Uric acid } \\
\hline Sarcoidosis patients & 23 & 6.05 & 2.141 & \multirow{2}{*}{2.255} & \multirow{2}{*}{$0.030 *$} \\
\hline Healthy controls & 14 & 4.64 & 1.197 & & \\
\hline \multicolumn{6}{|l|}{ Calcium } \\
\hline Sarcoidosis patients & 40 & 9.71 & 0.616 & \multirow{3}{*}{1.911} & \multirow{3}{*}{0.061} \\
\hline Healthy controls & 19 & 9.41 & 0.464 & & \\
\hline Healthy controls & 18 & 105.61 & 2.500 & & \\
\hline \multicolumn{6}{|l|}{$\mathrm{LDH}$} \\
\hline Sarcoidosis patients & 28 & 212.21 & 52.959 & \multirow{2}{*}{3.135} & \multirow{2}{*}{$0.003^{* *}$} \\
\hline Healthy controls & 18 & 176.39 & 23.410 & & \\
\hline \multicolumn{6}{|l|}{ ALP } \\
\hline Sarcoidosis patients & 28 & 75.00 & 26.980 & \multirow{2}{*}{1.347} & \multirow{2}{*}{0.187} \\
\hline Healthy controls & 9 & 62.00 & 17.804 & & \\
\hline \multicolumn{6}{|l|}{ CRP } \\
\hline Sarcoidosis patients & 44 & 6.77 & 9.993 & \multirow{2}{*}{0.755} & \multirow{2}{*}{0.453} \\
\hline Healthy controls & 19 & 4.61 & 11.319 & & \\
\hline
\end{tabular}

$\mathrm{LDH}$, lactate dehydrogenase; CRP, C-reactive protein. ${ }^{*} p<0.05 .{ }^{* *} p<0.01$.

\section{Results}

Seventy-three cases were included in this; $65.8 \%(n=$ 48) had been diagnosed with sarcoidosis and $34.2 \%$ ( $n=$ $25)$ were healthy controls; $58.9 \%(n=43)$ of all subjects were female and $41.1 \%(n=30)$ were male. Women and men accounted for $62.5 \%(n=30)$ and $37.5 \%(n=18)$ of the sarcoidosis patients, while in the control group 50\% $(n=13)$ of the cases were male and $50 \%(n=13)$ were female. The mean age of the entire study population was $42.11 \pm 12.29$ (20-67) years, while mean age of the patient and control groups was $45.21 \pm 11.90$ (23-67) and 36.16 \pm 10.953 (20-64) years, respectively.

Of all subjects, $49.30 \%(n=36)$ were smokers and $50.70 \%(n=37)$ had never smoked; smokers and those who had never smoked constituted $41.10 \%(n=20)$ and $58.30 \%(n=28)$ of the patient group and $46.15 \%(n=$
$12)$ and $38.40 \%(n=10)$ of the control group, respectively.

The staging of the patients according to Scadding staging was as follows: stage 0 patients $25 \%(n=12)$, stage 1 patients $35.4 \%(n=17)$, stage 2 patients $25 \%(n=12)$, stage 3 patients $12.5 \%(n=6)$, and stage 4 cases $2.1 \%(n=1)$. Of all the patients with sarcoidosis, $58.3 \%(n=28)$ had previously undergone steroid therapy, while $21.3 \%(n=10)$ were actively using steroid therapy while participating in the study. In addition, $4.2 \%(n=2)$ of patients were concomitantly receiving methotrexate treatment. Extrapulmonary involvement was present in $37.5 \%(n=18)$ of sarcoidosis patients, and eye, skin, and kidney involvement was present in $6.25 \%(n=3), 31.25 \%(n=15)$, and $8.3 \%(n$ $=4$ ) of the patients, respectively. Of all the patients, $2.08 \%$ $(n=1)$ had concomitant skin and eye involvement, whereas $4.16 \%(n=2)$ had both skin and kidney involvement. 
Table 2. Investigation of the differences in serum galectin-3 levels according to stages of sarcoidosis (independent samples $t$ test)

\begin{tabular}{lllllll}
\hline Stage of sarcoidosis & $N$ & Mean & SD & $F$ & $p$ value & Difference \\
\hline Stage 0 & 12 & 10.70 & 2.834 & & & \\
Stage 1 & 17 & 12.75 & 3.176 & 19.385 & $\mathbf{0 . 0 0 0 * * *}$ & $0.1-2$ \\
Stage 2 and higher & 19 & 19.39 & 5.440 & & & \\
Total & 48 & 14.87 & 5.567 & & & \\
\hline \multirow{2}{*}{$* * * * 0.001}$. & & & & & & \\
\hline
\end{tabular}

Table 3. Investigation of the relationship between serum galectin-3 levels and demographical, hematological, biochemical, and functional parameters

\begin{tabular}{|c|c|c|c|c|c|c|c|c|c|}
\hline Parameters & & Total & Sarcoidosis & $\begin{array}{l}\text { Healthy } \\
\text { controls }\end{array}$ & $\begin{array}{l}\text { Para- } \\
\text { meters }\end{array}$ & & Total & Sarcoidosis & $\begin{array}{l}\text { Healthy } \\
\text { controls }\end{array}$ \\
\hline \multirow[t]{2}{*}{ BMI } & $r$ & 0.143 & 0.135 & 0.056 & \multirow{2}{*}{ PLT } & $r$ & -0.023 & -0.133 & 0.078 \\
\hline & $p$ & 0.227 & 0.362 & 0.792 & & $p$ & 0.846 & 0.374 & 0.716 \\
\hline Smoking & $r$ & -0.004 & 0.161 & 0.111 & \multirow{2}{*}{ WBC } & $r$ & 0.372 & 0.412 & 0.072 \\
\hline$P / Y$ & $p$ & 0.972 & 0.275 & 0.597 & & $p$ & $0.001^{* *}$ & $0.004^{* *}$ & 0.738 \\
\hline \multirow[t]{2}{*}{ Blood glucose } & $r$ & 0.379 & 0.072 & 0.113 & \multirow{2}{*}{$\mathrm{RBC}$} & $r$ & 0.401 & 0.433 & 0.328 \\
\hline & $p$ & $0.003^{* *}$ & 0.628 & 0.592 & & $p$ & $0.001^{* *}$ & $0.002^{* *}$ & 0.118 \\
\hline \multirow[t]{2}{*}{ BUN } & $r$ & 0.262 & 0.349 & 0.272 & \multirow{2}{*}{$\mathrm{HGB}$} & $r$ & 0.235 & 0.312 & 0.312 \\
\hline & $p$ & $0.033^{*}$ & $0.027^{*}$ & 0.234 & & $p$ & $0.049^{*}$ & $0.033^{*}$ & 0.138 \\
\hline \multirow[t]{2}{*}{ Creatinine } & $r$ & 0.167 & 0.181 & 0.194 & \multirow{2}{*}{$\mathrm{HCT}$} & $r$ & 0.308 & 0.347 & 0.408 \\
\hline & $p$ & 0.181 & 0.230 & 0.412 & & $p$ & $0.009^{* *}$ & $0.017^{*}$ & $0.048^{*}$ \\
\hline \multirow[t]{2}{*}{ Uric acid } & $r$ & 0.315 & 0.143 & 0.232 & \multirow{2}{*}{ NLR } & $r$ & 0.352 & 0.266 & 0.105 \\
\hline & $p$ & 0.057 & 0.348 & 0.312 & & $p$ & $0.003^{* *}$ & 0.070 & 0.625 \\
\hline \multirow[t]{2}{*}{ Calcium } & $r$ & 0.097 & 0.230 & 0.233 & \multirow{2}{*}{ PLR } & $r$ & 0.047 & -0.127 & 0.151 \\
\hline & $p$ & 0.466 & 0.292 & 0.424 & & $p$ & 0.695 & 0.394 & 0.483 \\
\hline \multirow[t]{2}{*}{ AST } & $r$ & 0.069 & -0.110 & -0.176 & \multirow{2}{*}{ LYM } & $r$ & 0.074 & 0.273 & 0.036 \\
\hline & $p$ & 0.584 & 0.576 & 0.484 & & $p$ & 0.540 & 0.063 & 0.869 \\
\hline \multirow[t]{2}{*}{ ALT } & $r$ & 0.284 & -0.033 & 0.346 & \multirow{2}{*}{ LYM, \% } & $r$ & -0.291 & -0.180 & -0.087 \\
\hline & $p$ & $0.023^{*}$ & 0.834 & 0.124 & & $p$ & $0.014^{*}$ & 0.225 & 0.687 \\
\hline \multirow[t]{2}{*}{ LDH } & $r$ & 0.449 & 0.294 & 0.289 & \multirow{2}{*}{ MON } & $r$ & 0.346 & 0.406 & -0.309 \\
\hline & $p$ & $0.002^{* *}$ & 0.052 & 0.217 & & $p$ & $0.003^{* *}$ & $0.005^{* *}$ & 0.142 \\
\hline \multirow[t]{2}{*}{ ALP } & $r$ & 0.265 & 0.401 & 0.051 & \multirow{2}{*}{ MON, \% } & $r$ & 0.005 & 0.046 & -0.248 \\
\hline & $p$ & 0.112 & $0.034^{*}$ & 0.841 & & $p$ & 0.966 & 0.759 & 0.243 \\
\hline \multirow[t]{2}{*}{ CRP } & $r$ & 0.137 & 0.197 & 0.376 & \multirow{2}{*}{ NEU } & $r$ & 0.418 & 0.406 & 0.096 \\
\hline & $p$ & 0.283 & 0.315 & 0.318 & & $p$ & $0.000^{* * *}$ & $0.005^{* *}$ & 0.654 \\
\hline \multirow[t]{2}{*}{ ESR } & $r$ & 0.173 & 0.049 & 0.484 & \multirow{2}{*}{ NEU, \% } & $r$ & 0.302 & 0.214 & 0.108 \\
\hline & $p$ & 0.262 & 0.800 & 0.067 & & $p$ & $0.011^{*}$ & 0.149 & 0.614 \\
\hline
\end{tabular}

Smoking P/Y, smoking package/year; AST, aspartate transaminase; ALT, alanine transaminase; LDH, lactate dehydrogenase; CRP, C-reactive protein; ESR, erythrocyte sedimentation rate; WBC, white blood cells; RBC, red blood cell; HCT, hematocrit; PLT, platelet; NLR, NEU LYM ratio; PLR, platelets lymphocyte ratio. ${ }^{*} p<0.05$. ${ }^{* *} p<0.01$. ${ }^{* * *} p<0.001$.

An independent samples $t$ test showed that mean galectin-3 levels, age, BMI, blood urea nitrogen (BUN), uric acid, lactate dehydrogenase, and sedimentation values were significantly higher in the sarcoidosis patients than in the healthy controls (shown in Table 1). One-way ANOVA revealed a statistically significant difference in serum levels of galectin-3 between sarcoidosis disease stages $(p<0.05)$ (Table 2$)$. The mean levels of serum ga- lectin-3 in the sarcoidosis patients at stage 2 or higher were significantly higher than those in patients at stage 0 or 1 . The independent samples $t$ test revealed no statistically significant differences in the mean levels of serum galectin-3 between the patients with and without a concomitant disease, between smokers and nonsmokers, between patients receiving and not receiving active treatment, and between patients with and without extrapul-
62

Med Princ Pract 2022;31:59-65 DOI: $10.1159 / 000521467$
Kara/Tural Onur/Nedime Sokucu/Kahya/ Ozdemir/Ademoglu 
monary involvement $(p>0.05)$. Compared to the healthy controls, a significantly stronger relationship was observed between the mean serum galectin-3 levels and the serum BUN, MON and NEU count, leukocyte and erythrocyte count, and hemoglobin (HGB) and hematocrit values in the sarcoidosis patients (Table 3 ).

\section{Discussion}

Sarcoidosis is a systemic granulomatous disease with unknown etiology and progresses with multi-systemic involvement. With a devious onset from asymptomatic bilateral hilar adenopathy to multiple extrapulmonary lesions, the disease may result in progressive fibrosis of the lungs and other organs. Prognosis and disease course may be associated with its mode of onset and its extent [1-3].

In the present study, we found that serum levels of galectin-3 were significantly higher in the sarcoidosis patients than in the healthy controls and that the levels were particularly elevated in patients at stage 2 or higher (characterized by parenchymal involvement), when compared to patients at stage 0 and 1 . On the other hand, extrapulmonary involvement, smoking, age, or BMI was not significantly associated with serum galectin-3 levels.

In vitro studies investigating galectin- 3 have demonstrated that the absence of galectin-3 may improve fibrotic response in the kidneys, liver, and lungs $[15,16]$. Previous studies have also reported increased levels of galectin-3 in diseases progressing with fibrosis, such as cirrhosis and idiopathic pulmonary fibrosis [13-17]. A previous study reported that galectin-3 levels were elevated in sarcoidosis patients with cardiac involvement when compared to the controls [18]. Another study reported that serum galectin-3 levels were similar in sarcoidosis patients and control groups [19]. These data indicate that galectin-3 may result in fibrosis in several organs. In the present study, we investigate the role of galectin- 3 in the staging and severity of sarcoidosis that progresses with fibrosis.

Consistent with the findings of previous studies [1418 ], galectin-3 levels were found to be significantly higher in sarcoidosis patients than in the healthy controls. While galectin-3 levels were compared based on the radiological stages of sarcoidosis, the levels were found to be significantly higher in patients at stage 2 or higher, progressing with parenchymal involvement, when compared to the healthy controls, as well as patients with stage 0 or 1 sarcoidosis who were in remission, and who were being followed without treatment. This increase in galec- tin-3 levels indicates that fibrosis developed as soon as parenchymal involvement occurred. The detection of high levels of galectin-3 prior to a radiological diagnosis of stage 2 can indicate disease progression before radiology and may help with early diagnosis, and the consideration of alternative treatments before the full development of pulmonary fibrosis.

We also observed that extrapulmonary involvements in such organs as the eye, kidney, and skin did not significantly affect galectin-3 levels in sarcoidosis patients. Similarly, a previous study reported elevated serum galectin-3 levels in patients with pulmonary sarcoidosis when compared to the control group but did not point to a significant difference in galectin-3 levels between patients with and without cardiac involvement [18]. The present study showed that galectin-3 levels in the sarcoidosis patients were not significantly affected by smoking status, age, gender, BMI, creatinine, serum calcium, lactate dehydrogenase, sedimentation, angiotensin-converting enzyme, FEV1\%, FVC\%, 24-h urine calcium, NEU, LYM ratio, or thrombocyte LYM ratio. A previous study identified a significant relationship between serum levels of galectin-3 and age and gender, found the levels to be higher in women than men, and were observed to increase with age, whereas we identified no such relationships in the present study [20]. The same study also reported significant relationships between galectin-3 levels and BMI, kidney function tests, DM, C-reactive protein, and smoking status, while no such relationships were identified to any significant degree between galectin- 3 levels and these parameters in the present study [20]. Similarly, a study performed in 2007 reported significantly elevated levels of serum galectin-3 in bronchoalveolar lavage samples obtained from patients with diseases progressing with pulmonary fibrosis, particularly in patients with idiopathic pulmonary fibrosis and interstitial lung disease associated with connective tissue disorders. Furthermore, the galectin-3 levels in patients with interstitial lung diseases associated with connective tissue disorders who underwent steroid treatment were also found to be mildly decreased, although this decrease was not statistically significant in that study, contradicting the findings of the present study [14]. This may be attributed to the inclusion of a healthy control group and sarcoidosis patients with normal renal functions in the present study.

Previous studies have also reported a relationship between high serum levels of galectin-3 and prognoses of acute and chronic heart failure, pulmonary hypertension, and acute coronary syndrome $[20,21]$. This suggests that serum galectin-3 may also be used as a marker of prognosis 
in sarcoidosis patients. The short duration of our study prevented the collection of a sufficient amount of data for the investigation of the relationship between serum galectin-3 levels and prognosis in sarcoidosis patients. We believe that future prospective studies will better establish the relationship between serum galectin-3 levels and disease prognosis.

The results of the present study reveal that HGB, hematocrit, red blood cell, white blood cells, MON and NEU values, and BUN levels have a positive effect on serum galectin-3 levels in sarcoidosis patients, although contrary to our findings, a previous study involving patients with heart failure found no significant relationship between HGB and serum galectin-3 levels [22]. Previous studies have identified a negative relationship between HGB and inflammation [23]. We believe that the differences between our findings and those of previous studies may be attributed to the fact that the majority of patients in the present study were in a stable phase of the disease or were in regression.

Elevated alkaline phosphatase (ALP) levels are detected in up to 33 percent of sarcoidosis patients, and ALP is superior to gamma-glutamyl transferase in terms of showing liver involvement. In this study, ALP levels were not significantly different between the healthy control group and the sarcoidosis patients, although ALP levels were found to be associated with galectin-3 levels in the sarcoidosis patients. Galectin-3 levels increase in the presence of acute and chronic inflammation. Like galectin-3, ALP is a biomarker that is used as a marker of inflammation [24]. The relationship between galectin-3 and ALP levels can be explained by the fact that the levels of both markers increase in the presence of inflammation.

Platelet distribution width (PDW) is associated with thrombocyte activation and has been shown to play a role in thrombosis, and to be elevated in the presence of bone marrow involvement and inflammation $[25,26]$. In the present study, PDW was significantly higher in the sarcoidosis patients when compared to the healthy controls. Moreover, a negative relationship was identified between PDW and galectin-3 levels in sarcoidosis patients, whereas a positive association was found between these 2 parameters in the control group. In the light of these data, it may be argued that pulmonary fibrosis may develop in sarcoidosis patients with low PDW values, although further studies are required to confirm this hypothesis.

We aimed to use galectin-3 in the prognosis or staging of sarcoidosis but not in the diagnosis of sarcoidosis fibrosis and fibrotic process is seen in advanced stages of sarcoidosis and fibrosis is not a specific condition of the disease. Our findings demonstrating that galectin-3 levels were significantly elevated in sarcoidosis patients at stage 2 or higher with parenchymal involvement suggest that serum galectin-3 can be used to assess the staging and severity of sarcoidosis. While several parameters have been investigated for the assessment of response to treatment and progression of sarcoidosis, there is currently no universally accepted and routinely used parameter for either of these purposes. Despite being a benign disease in general, sarcoidosis is still is a matter of concern due to the challenges faced in its diagnosis, its economic burden on health expenditure, and its consequences in terms of loss of workforce. We suggest that serum galectin- 3 could be a useful parameter in sarcoidosis for several reasons, including its ease of use, its amenability for rapid testing and its ability to identify fibrosis earlier than radiology, and the consequent avoidance of radiation exposure. That said, there are several limitations to our study, including the low number of patients at stage 3 and 4. Furthermore, this study was not designed as a controlled progressive study for the monitoring of patients with diseases progressing with pulmonary fibrosis. In addition, only a snapshot of galectin-3 levels was obtained in the study, and therefore, serum galectin- 3 levels during the course of disease were not compared in sarcoidosis patients taking into account progression status. Accordingly, there is a need for prospective studies involving larger patient groups to investigate the routine use of serum galectin-3 in sarcoidosis patients and clarify its relationship with other parameters affecting disease course.

\section{Conclusion}

There are currently no parameters that can be used as a marker of progression and disease activity in sarcoidosis. In the present study, we found that serum levels of galectin-3 were significantly elevated in sarcoidosis patients when compared to healthy controls and that levels were significantly increased particularly in patients at stage 2 or higher, associated with parenchymal involvement. Serum galectin-3 levels may be used in the monitoring of sarcoidosis, being a simple and rapid approach that avoids radiation exposure and reveals pulmonary fibrosis prior to radiological staging.

\section{Acknowledgment}

The authors thank the Turkish Respiratory Society for editing and statistics. This work was presented at the 39th Respiratory Congress, Turkish Respiratory Society, Izmir, Turkey, April 1417, 2017.
Med Princ Pract 2022;31:59-65 DOI: $10.1159 / 000521467$
Kara/Tural Onur/Nedime Sokucu/Kahya/ Ozdemir/Ademoglu 


\section{Conflict of Interest Statement}

The authors have no conflicts of interest to declare.

\section{Funding Sources}

There is no funding source. The researchers conducted the research with their own budgets.

\section{Author Contributions}

Kaan Kara contributed to idea and design of the research, patient selection, data collection, interpretation of statistics and results, and manuscript writing; Sinem Nedime Sokucu and Seda
Tural Onur contributed to design of the study and critical revision for intellectual content. Cengiz Ozdemir and Ozlem Kahya contributed to data collection; Evin Ademoglu contributed to laboratory work.

\section{Data Availability Statement}

The data of this study are not publicly available as they contain information that could compromise the privacy of research participants but are available from K.K. (kaankara3643@yahoo.com) upon reasonable request.

\section{References}

1 Akkaynak S. Sarcoidosis. Tuberc Toraks Med. 1953;1:236-50.

2 Hutchinson J. Statement on sarcoidosis. Joint statement of the American Thoracic Society (ATS), the European Respiratory Society (ERS) and the World Association of Sarcoidosis and Other Granulomatous Disorders (WASOG) adopted by the ATS Board of Directors and by the ER. Am J Respir Crit Care Med. 1999;160:55.

3 Musellim B, Kumbasa OO, Ongen G, Çetinkaya E, Turker H, Uzaslan E, et al. Epidemiological features of Turkish patients with sarcoidosis. Respir Med. 2009;103:907-12.

4 Çağatay T, Bilis S, Yanardağ H, Gülbaran Z, Papila Ç. The immunoglobulin and complement levels in the active pulmonary sarcoidosis. Kobe J Med Sci. 2003;49:99-106.

5 Planck A, Eklund A, Grunewald J. Markers of activity in clinically recovered human leukocyte antigen-DR17-positive sarcoidosis patients. Eur Respir J. 2003;21:52-7.

6 Alessandro M, Bergantini L, Cameli P, Vietri L, Lanzarone N, Alonzi V, et al. Krebs von den Lungen- 6 as a biomarker for disease severity assessment in interstitial lung disease: a comprehensive review. Biomark Med. 2020;14(8) 665-74.

7 Bennett D, Cameli P, Lanzarone N, Carobene L, Bianchi N, Fui A, et al. Chitotriosidase: a biomarker of activity and severity in patients with sarcoidosis. Respir Res. 2020;21(1).

8 Kim H, Lee J, Hyun JW, Park JW, Joo HG, Shin T. Expression and immunohistochemical localization of galectin-3 in various mouse tissues. Cell Biol Int. 2007;31:655-62.

9 Barondes SH, Cooper DN, Gitt MA, Leffler H. Galectins. Structure and function of a large family of animal lectins. J Biol Chem. 1994; 269:20807-10.
10 Sato S, Hughes RC. Regulation of secretion and surface expression of Mac-2, a galactoside-binding protein of macrophages. J Biol Chem. 1994;269:4424-30.

11 Almkvist J, Karlsson A. Galectins as inflammatory mediators. Glycoconj J. 2002;19:57581.

12 Ikemori R, Machado CML, Furuzawa KW Nonogaki S, Osinaga E, Umezawa K, et al. Galectin-3 up-regulation in hypoxic and nutrient deprived. PLoS One. 2014 Nov 4;9(11): e111592.

13 Sun H, Jiang H, Eliaz A, Kellum JA, Peng Z, Eliaz I. Galectin-3 in septic acute kidney injury: a translational study. Crit Care. 2021 Mar 18;25(1):109.

14 Nishi Y, Sano H, Kawashima T, Okada T, Kuroda T, Kikkawi K, et al. Role of galectin-3 in human pulmonary fibrosis. Allergol Int. 2007; 56:57-65.

15 Henderson NC, Mackinnon AC, Farnworth SL, Poirier F, Russo FP, Iredale JP, et al. Galectin-3 regulates myofibroblast activation and hepatic fibrosis. Proc Natl Acad Sci U S A. 2006;103:5060-5.

16 Mackinnon AC, Gibbons MA, Farnworth SL, Leffler H, Nilsson UJ, Delaine T, et al. Regulation of transforming growth factor- $\beta 1$-driven lung fibrosis by galectin-3. Am J Respir Crit Care Med. 2012;185:537-46.

17 d'Alessandro M, Vita ED, Bergantini L, Mazzei MA, Valvasone S, Bonizzoli M, et al. Galactin-1, 3 and 9: potential biomarkers in idiopathic pulmonary fibrosis and other interstitial lung diseases. Respir Physiol Neurobiol. 2020 Nov; $282: 103546$.

18 Kul S, Ozcelik HK, Uyarel H, Karakus G, Guvenc TS, Yalçınsoy M, et al. Diagnostic value of strain echocardiography, galectin-3, and tenascin- $\mathrm{C}$ levels for the identification of patients with pulmonary and cardiac sarcoidosis. Lung. 2014;192:533-42.
19 Kobak S, Akyildiz M, Gokduman A, Atabay T, Vural H. Serum galectin-3 and TGF-beta levels in patients with sarcoidosis. Reumatol Clin. 2021 Dec;17(10):562-5.

20 De Boer R, Van Veldhuisen DJ, Gansevort RT, Muller Kobold AC, Van Gilst WH, Hillege HL, et al. The fibrosis marker galectin-3 and outcome in the general population. J Intern Med. 2012;272:55-64.

21 Van Der Velde AR, Gullestad L, Ueland T, Aukrust P, Guo Y, Adourian A, et al. Prognostic value of changes in galectin-3 levels over time in patients with heart failure: data from CORONA and COACH. Circ Heart Fail. 2013;6(2):219-26.

22 Gullestad L, Ueland T, Kiekshus J, Nymo SH, Hulthe J, Muntendam P, et al. The predictive value of galectin-3 for mortality and cardiovascular events in the Controlled Rosuvastatin Multinational Trial in Heart Failure (CORONA). Am Heart J. 2012;164:878-83.

23 Wang $\mathrm{CH}$, Cheng ML, Liu MH, Kuo LT, Shio MS. Metabolic profile provides prognostic value better than galectin-3 in patients with heart failure. J Cardiol. 2017;70(1):92-8.

24 Macciò A, Madeduce C, Massa D, Mudu MC, Lusso MR, Gramignano G, et al. Hemoglobin levels correlate with interleukin-6 levels in patients with advanced untreated epithelial ovarian cancer: role of inflammation in cancer-related anemia. Blood. 2005;106:362-7.

25 Henderson NC, Sethi T. The regulation of inflammation by galectin-3. Immunol Rev. 2009;230:160-71.

26 Elzey BD, Tian J, Jensen RJ, Swanson AK, Lees JR, Lentz SR, et al. Platelet-mediated modulation of adaptive immunity: a communication link between innate and adaptive immune compartments. Immunity. 2003;19:9-19. 